\title{
ASSESSMENT OF PLANT FUNCTIONAL TYPES IN TROPICAL ARID AND SEMI-ARID ECOSYSTEMS OF INDIA USING REMOTE SENSING DATA AND GIS
}

\author{
C. Sudhakar Reddy, P. Hari Krishna and M.S.R. Murthy \\ Forestry \& Ecology Division, National Remote Sensing Centre, ISRO, Balanagar, Hyderabad- 500 625, India \\ E-mail: drsudhakarreddy@gmail.com
}

\begin{abstract}
:
Tropical ecosystems undergo changes caused by season, climate or multiple anthropogenic impacts. Such changes may cause gradual or rapid shifts from one state to another. There has been a focus on functional classifications of plants to find tools for monitoring and assessing species status in changing environments. It has been recognised that plant biological characteristics can be related to their response to predominant environmental factors and interactions between other organisms. These findings have resulted in a search for plant functional types (PFTs) that are user-defined groups of species with similar response to environmental resources and disturbance associated to common biological traits. Now, identification of plant functional types is priority area in the climate change research. Satellite Earth observation data is an important tool in providing considerable information on extracting PFT information at global and regional levels. From the modelling perspective, some of the current needs are the refinement of processes that govern community assembly, such as natural and anthropogenic disturbances. PFTs used in large-scale models are insufficient to represent the diversity of responses in natural plant communities. The currently available MODIS PFT map was generated by re-labeling the IGBP land cover type classes. However, the error magnitudes of the MODIS PFT product and their spatial and temporal distributions have not been fully characterized. Remotely sensed derived information of the phenology, community composition and vegetation structure are the key inputs to integrate with the variability in precipitation and temperature to map the spatial distribution of Plant functional types. PFTs allows accurate representation of the land surface by separately specifying the composition and structure of PFTs within a grid cell. Very little research efforts are discernible in India that explicitly address the PFTs.
\end{abstract}

In the present study five natural vegetation systems were studied along arid, semi-arid and sub-humid regions of Indian Desert and Aravallis of Rajasthan, India, with a distance of $600 \mathrm{~km}$. The annual precipitation decreases from as high as $1600 \mathrm{~mm}$ in the south to 700 $\mathrm{mm}$ in the north-east and $100 \mathrm{~mm}$ in the west. The study is based on the integrated approach of remote sensing, GIS, and phytosociology. In the step 1, vegetation type map was prepared using multi-season IRS P6 LISS III data. Screening of plant traits was done based on field observations and literature. Phytosociological data pertains to 500 sample plots was used to identify plant functional traits of 900 species at morphological level. The vegetation classification scheme at regional level identified thorn forest, dry deciduous forest, broad leaved forest, woodland, shrubland and grasslands. Five plant traits selected in the study were significant for tropical environments. Attributes such as leaf size, leaf texture, spinescence, stem diameter and bark consistency were categorized systematically. Ordination analysis was carried out across the environmental gradient. Plant functional traits were measured on 20 individuals per species at each site. Environmental information was integrated to identify plant trait response. The spatial trends in PFTs were analysed and compared across the vegetation types, along the gradient of land surface temperature, climate, elevation and time integrated NDVI. Results established the occurrence of recurrent patterns of association among selected plant traits. Functional response groups were identified by summarizing results and relating them to individual species. Finally phytoclimatic map was prepared to represent spatial distribution of PFTs.

The species with of functional traits of representing microphylls, sclerophyll, rough bark, spinescence and therophyte are demarcated as drought tolerant traits. Drought Intolerant PFTs are represented by macrophyll, malacophyll, smooth Bark, non spinescent stems and leaves. Biological spectrum of Desert national park, Jaisalmer and and Ajmer represents therophytic climate (below 600mm rainfall) while Sariska and Mt. Abu are of therophanerophytic $(>600 \mathrm{~mm}$ rainfall). The higher proportion of microphylls and therophytes are distributed within tropical arid environments. The dominance of the drought tolerant PFTs increased towards west while the dominance of the drought intolerant PFTs increased in southern and northern parts of Rajasthan. Detecting the change of spatial characteristics of plant species under precipitation gradients would be helpful to study species dynamics in a large region under environmental gradient. On the basis of easily-measured plant traits and phytosociological surveys, the present approach summarized the complexity of tropical vegetation into a relatively few PFTs. The present study open up new possibilities for understanding plant trait and environment relationship and developing PFT classification in tropical regions. 